\title{
IDIOPATHIC PULMONARY HEMOSIDEROSIS AS A DIFFERENTIAL DIAGNOSIS OF AUTOIMMUNE DISEASES: CASE REPORT
}

Danielly Dantas Pimentel ${ }^{1, \star}$, Gizelle Gouvea Rezende ${ }^{1}$, Gustavo Roberto Lourenço1 ${ }^{1}$ Iane Tamara Dondé ${ }^{1}$, Juliana de Jesus Boscolo ${ }^{1}$, Luciana Akita ${ }^{1}$, Viviane Alves Costa ${ }^{1}$, Viviane Queiroz de Oliveira Maia' ${ }^{1}$ Taisa Morete da Silva ${ }^{1}$

1.Faculdade de Medicina de São José do Rio Preto, São José do Rio Preto (SP), Brazil.

*Corresponding author: daniellydantas2011@gmail.com

\section{BACKGROUND}

Diffuse alveolar hemorrhage (DAH) can appear in multiple contexts, such as immunological or infectious diseases, coagulation disorders and cardiac and renal pathologies. Clinically, it manifests itself through hemoptysis or dry cough only, chest pain and dyspnea. When there is no underlying cause that explains recurring episodes of DAH, the disease is known as idiopathic pulmonary hemosiderosis (IPH). The exact incidence and prevalence of IPH are unknown. In adults, most cases occur between 20 and 40 years of age, although it can occur at any age.

\section{CASE REPORT}

A 24-year-old female patient, previously healthy, denies having used any medication continuously until 2017, when she was hospitalized with hemoptysis, dyspnea, weight loss and asthenia. On this occasion, a pulmonary parenchymal infiltrate was found on chest radiography, as well as microcytic hypochromic anemia of undefined etiology. She had normal autoantibodies and serological tests along with normal lung biopsy. She was discharged and started to follow up on an outpatient basis until she lost follow-up. The patient returned in 2020, presenting hemoptysis and dyspnea once again. According to her, she had remained asymptomatic for the past 3 years. Again, serological and autoantibodies tests were normal. She presented microcytic hypochromic anemia, positive direct Coombs test, high LDH levels and high reticulocyte count, in addition to chest tomography showing ground-glass opacities and thickening of the interlobular septa affecting both lungs. Lung biopsy was performed and hemosiderin was observed in alveolar macrophages.

\section{CONCLUSION}

The reported case shows a young patient with DAH and microcytic hypochromic anemia on top of altered hemolysis tests. A diagnostic hypothesis of IPH was suggested, which, despite being a rare disease, would explain the recurrent clinical presentation of alveolar hemorrhage happening in the absence of other causes of intrapulmonary bleeding or extrapulmonary involvement. The pathogenesis of the disease is obscure. Most theories refer to autoimmune factors since HPI resembles other autoimmune diseases, such as systemic lupus erythematosus, and because the use of immunosuppressants improves the patient's clinical condition. Hemolytic anemia associated with positive direct Coombs test can also be found in IPH, suggesting an immunological basis and often delaying the diagnosis, which can be confirmed only after other causes have been ruled out and once the results of the anatomopathological examination of lung biopsy show hemosiderin-containing macrophages (siderophages). The reported case is an example of idiopathic pulmonary hemosiderosis, since it has been shown not to be caused by other underlying diseases and because the patient has both the clinical picture and the biopsy results consistent with the hypothesis. 